\title{
A NOTE ON INTEGRAL GEOMETRY IN THE INVERSIVE PLANE
}

\author{
JAY P. FILLMORE
}

\begin{abstract}
The density for circles in the Euclidean plane, which is invariant under the group of similitudes, is in fact invariant under the inversive group. The fundamental invariants of the inversive plane, angle and inversive distance, can be obtained from the measures of certain sets of circles.
\end{abstract}

1. Circles. The set of circles in the Euclidean plane has density, taken in absolute value,

$$
d C=d a d b d r / r^{3},
$$

where $a$ and $b$ are the rectangular coordinates of the center of the circle and $r$ is its radius. This density is invariant under the group of similitudes of the plane [2, p. 161]. But, it is furthermore invariant under the inversive group of the plane.

The inversive group, or conformal group, of the plane is generated by inversions in circles and contains all similitudes; it is also generated by similitudes together with the single additional transformation

$$
x^{\prime}=x /\left(x^{2}+y^{2}\right), \quad y^{\prime}=y /\left(x^{2}+y^{2}\right),
$$

inversion in the unit circle. It suffices to verify that $d C$ is invariant under this inversion.

Inversion in the unit circle sends the circle with center $(a, b)$ and radius $r$ into the circle with center $\left(a^{\prime}, b^{\prime}\right)$ and radius $r^{\prime}$, where

$$
a^{\prime}=F a, \quad b^{\prime}=F b, \quad r^{\prime}=|F| r, \quad \text { with } F=\left(a^{2}+b^{2}-r^{2}\right)^{-1} .
$$

Direct calculation gives

$$
d a^{\prime} d b^{\prime} d r^{\prime}=F^{2}\left|F+a \frac{\partial F}{\partial a}+b \frac{\partial F}{\partial b}+r \frac{\partial F}{\partial r}\right| d a d b d r .
$$

Since $F$ is homogeneous of degree -2 in $a, b, r$, Euler's identity gives $-F$ for the expression in absolute values. From $d a^{\prime} d b^{\prime} d r^{\prime}=|F|^{3} d a d b d r$ and $r^{3}=|F|^{3} r^{3}$, one obtains the invariance of $d C$.

There is a deeper reason that the density $d C$ is invariant under the inversive group. The inversive plane, with the point at infinity adjoined, is a sphere; the interior of the sphere carries a definite metric of constant negative curvature, and conformal transformations of the sphere correspond to isometries of the interior. Now, the exterior of the sphere carries an indefinite metric of constant negative curvature and the isometries of the exterior correspond to those of the interior. An exterior point, by intersecting its polar plane with the sphere, represents a circle in

Received by the editors November 22, 1978.

AMS (MOS) subject classifications (1970). Primary 50D45, 60D05. 
the inversive plane. Conformal transformations correspond to isometries of the exterior, and $d C$ is the volume element of this indefinite metric.

The nature of the expression for $d C$ shows that sets of circles having arbitrarily small radius cannot be expected to have finite measures, and consequently there is no analog of Crofton's Theorem [2, p. 31]. The closest one has to Crofton's Theorem is $\int n d C=4 L / r_{0}$, where $L$ is the length of a fixed curve, $n$ is the number of intersections of a variable circle with the fixed curve, and the integral is extended over circles having radius at least $r_{0}$. This is a consequence of Poincaré's Formula [2, p. 112]. But, this formula belongs to the geometry of similitudes and not to inversive geometry.

A set of circles whose radii are not too small can be expected to have finite measure; more precisely, a set of circles for which $r /\left|a^{2}+b^{2}-r^{2}+1\right|$ is bounded away from zero will have finite measure. Furthermore, it is possible to determine the fundamental invariants of the inversive plane, angle and inversive distance, in terms of such sets of circles.

2. Formulas. For two circles of radii $a$ and $b$ and centers separated by distance $c$, $\left|\left(a^{2}+b^{2}-c^{2}\right) / 2 a b\right|$ gives the cosine of the angle between the circles in case they intersect, and $\cosh \delta$, where $\delta$ is the inversive distance between the circles, in case they do not intersect [1, p. 129]. Angle and inversive distance are invariant under the inversive group. If the circles are concentric, one has $\delta=|\log (b / a)|$.

The region between two nonintersecting circles will mean: the region exterior to one and interior to the other in case one of the circles lies inside the other, and the region exterior to both in case each circle lies outside the other.

Formula 1. The measure of all circles lying in the region between two given nonintersecting circles is

$$
\int d C=\pi\left(\delta-2 \tanh \frac{\delta}{2}\right)
$$

where $\delta$ is the inversive distance between the given circles.

The function $\delta-2 \tanh (\delta / 2)$ is invertible, so inversive distance can be determined in terms of measures of sets of circles.

A circle which meets all three sides of a triangle lies in the region between the incircle and the circumcircle of the triangle. The inversive distance between these two circles is given by $\sinh (\delta / 2)=\frac{1}{2} \sqrt{r / R}[1, \mathrm{p} .131]$. Consequently, one can write a formula for the measure of the set of circles which meet all three sides of a triangle in terms of the inradius $r$ and the circumradius $R$.

The analog of Formula 1 for angles is somewhat more complicated. Given two intersecting circles, construct two nonintersecting auxiliary circles both of which are orthogonal to both of the given circles.

Formula 2. The measure of all circles which meet both of two given intersecting circles and which meet the first but not the second auxiliary circle is

$$
\int d C=4 \delta \csc \omega
$$


where $\omega$ is the angle between the given circles, and $\delta$ is the inversive distance between the auxiliary circles.

Consequently, angles can be determined in terms of measures of sets of circles.

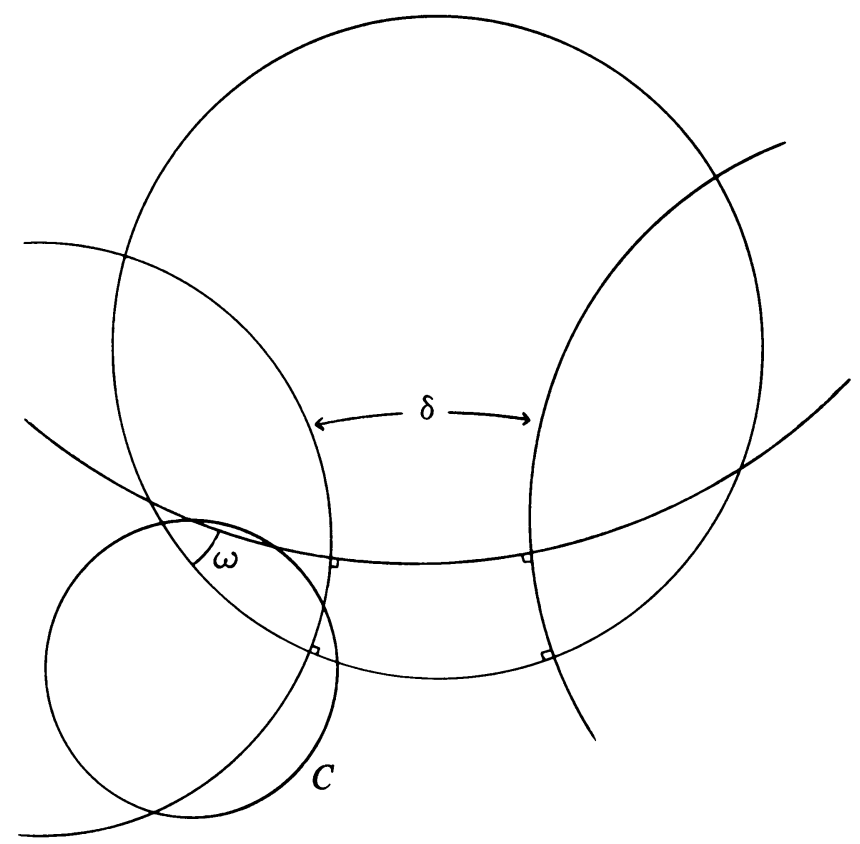

3. Proofs. Formula 1. Any two nonintersecting circles can be inverted into concentric circles [1, p. 121]. Since the quantities in the formula are invariant under inversion, it suffices to verify it in this special case. Let the concentric circles have center at the origin and radii $a$ and $b, a<b$; let $\rho$ and $\theta$ be the polar coordinates of the center of the variable circle $C, r$ its radius. Then

$$
\int d C=\iiint_{\substack{r>a+\rho \\ b>\rho+r \\ 0<\theta<2 \pi}} \frac{\rho d \rho d \theta d r}{r^{3}}
$$

The first two inequalities insure that $C$ lies in the region between the concentric circles. It is elementary to evaluate this integral and obtain

$$
\int_{0}^{(b-a) / 2}\left(\int_{a+\rho}^{b-\rho} \frac{\rho}{r^{3}} d r\right) d \rho \cdot \int_{0}^{2 \pi} d \theta=\pi\left(\log \frac{b}{a}-2 \frac{b-a}{b+a}\right) .
$$

Since $\delta=\log (b / a)$, one has Formula 1 .

Formula 2. Any two intersecting circles can be inverted into intersecting lines. It suffices to verify the formula in this special case. Take the point of intersection of the lines as origin. The auxiliary circles have their centers at the origin; let $a$ and $b$, $a<b$, be their radii. Let $\rho$ and $\theta$ be the polar coordinates of the center of the variable circle $C, r$ its radius. The angle between the lines is $\omega$. Then 


$$
\int d C=\iiint_{\substack{a<\rho+r<b \\ \rho|\sin \theta|<r, \rho|\sin (\omega-\theta)|<r \\ 0<\theta<2 \pi}} \frac{\rho d \rho d \theta d r}{r^{3}}
$$

The first double inequality insures $C$ meets the circle of radius $a$ but not the circle of radius $b$; the second pair of inequalities insures that $C$ meets both lines. Set

$$
m(\theta)=\operatorname{Max}(|\sin \theta|,|\sin (\omega-\theta)|) ;
$$

the integral becomes

$$
\int_{0}^{2 \pi}\left[\iint_{\substack{a<\rho+r<b \\ \rho m(\theta)<r}} \frac{\rho}{r^{3}} d \rho d r\right] d \theta .
$$

The inner double integral can be evaluated using the variables $u=\rho+r$ and $r$; one obtains

$$
\int_{a}^{b}\left(\int_{(m /(1+m)) u}^{u} \frac{u-r}{r^{3}} d r\right) d u=\frac{1}{2 m(\theta)^{2}} \log \frac{b}{a} .
$$

The desired integral becomes

$$
\frac{1}{2} \log \frac{b}{a} \int_{0}^{2 \pi} \frac{1}{m(\theta)^{2}} d \theta .
$$

Now, $m(\theta)=\sin \theta$ for $\omega / 2<\theta<(\omega+\pi) / 2$ and the graph of $m(\theta)$ consists of four regions congruent to this portion. Hence,

$$
\int_{0}^{2 \pi} \frac{1}{m(\theta)^{2}} d \theta=4 \int_{\omega / 2}^{(\omega+\pi) / 2} \csc ^{2} \theta d \theta=8 \csc \omega .
$$

Finally, $\delta=\log (b / a)$ gives Formula 2 .

\section{REFERENCES}

1. H. S. M. Coxeter and S. L. Greitzer, Geometry revisited, Random House, New York, 1967.

2. L. A. Santaló, Integral geometry and geometric probability, Addison-Wesley, Reading, Mass., 1976.

Department of Mathematics, University of California at San Diego, la Jolla, California 92093 